With $n=2$, this solution would be identical with that for a wedge of constant thickness. However, this would necessitate a fictitious $\nu$ of $3 / 2$.

(c) Uniform Pressure on Edge, Fig. 3. The stress function required hete is

$$
\phi=r^{3}(A+B \sin n \theta+C \cos n \theta+D \theta) ; n^{2}=(7-2 \nu)
$$

whence

$$
\begin{gathered}
h \sigma_{r}=3 A r+\left(3-n^{2}\right) B r \sin n \theta+\left(3-n^{2}\right) c r \cos n \theta+3 D r \theta \\
h \sigma_{\theta}=6 A r+6 B r n \sin n \theta+6 C r n \cos n \theta+6 D r \theta \\
h \tau_{r \theta}=-2 B r \cos n \theta+2 C r \sin n \theta-2 D r ; h=h_{0} r
\end{gathered}
$$

From the boundary conditions and Fig. 3

$$
\begin{array}{llll}
\theta & =0, & h \sigma_{\theta}=-h_{\mathrm{n}} p r, & \tau_{r \theta}=0 \\
\theta=2 \alpha, & h \sigma_{\theta}=0, & \tau_{r \theta}=0
\end{array}
$$

we obtain

$$
\begin{aligned}
A & =-p h_{0}(1-\cos 2 n \alpha-2 n \alpha \sin 2 n \alpha) / N \\
B n & =-D=p h_{0} n \sin 2 n \alpha / N \\
C & =-p h_{0}(1-\cos 2 n \alpha) / N
\end{aligned}
$$

where $N=6(2-2 \cos 2 n \alpha-2 n \alpha \sin 2 n \alpha)$. The stresses, obtained by substituting lispuations (10) in (9), are seen to be independent of $r$.
NOTICE: This material may be protected by copyright law (title 17 U.S. Code)

\section{Minimization of Spring Torque in Flexible Connections to Instruments}

\section{T. VREELAND, Jr. ${ }^{1}$}

Precision electromechanical measuring instruments of various kinds often require establishing electrical connection to the suspended part of the instrument, with minimum introduction of disturbing torques. Requirements are especially demanding in the case of gyroscopes. Gyroscopes of high-precision type, as used in inertial-guidance systems and in advanced flight-control systems, generally take the form of a gimbal member which contains the rotor and which is supported relative to the case by some form of minimum-friction bearings. Usually, at least three electrical connections are required (for the polyphase rotor drive motor), which take the form of wires or ribbons extending from the case to the gimbal member. Disturbing-torque levels about the gimbal axis or axes must be kept to extremely low values; the total tolerable disturbing torque from all sources whatever is often of the order of one dyne-cm or less. Special attention must be given to the electrical lead-in connections $(a)$ to minimize mechanical hysteresis effects, which result in erratic bias changes, and $(b)$ to minimize spring rate. While spring coercion is a conservative type of force, it nevertheless must be kept very small, otherwise a shift of null of the pickoff which detects gimbal displacement will result in a steady spring bias.

The present note is restricted to the optimization of the electrical lead-in configuration from the point of view of minimizing

\footnotetext{
1 Associute Professor of Mechanical Engineering, California Institute of 'Technology, Pasadena, Calif.

Manuscript received by AsMF Applied Mechanics Division, February 18,1959 .
}

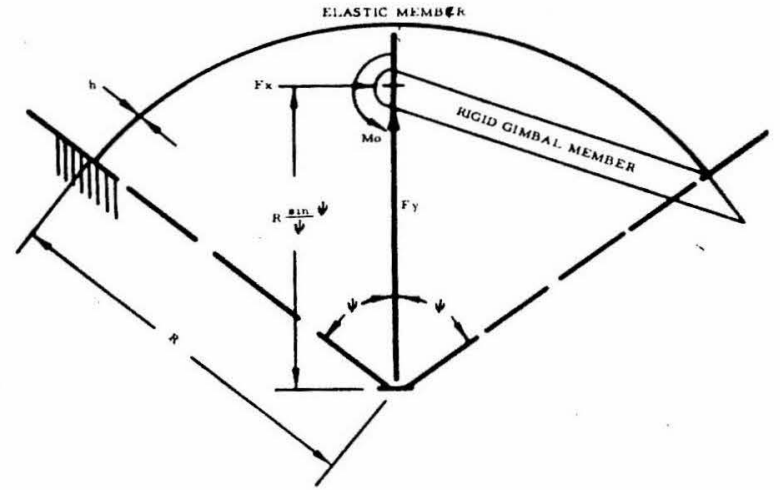

F:g. I Application of forces to an elastic member by a gimbal member pivoted at the elastic center

spring rate. In general, the axis of the gimbal member is inaccessible, so that electrical connections will take the form of curved wires or ribbons, bypassing the axis.

Consider the elastic member with flexural rigidity $E I$, in the form of a circular are of radius $R$, and length $2 R \psi$, with one end fixed to a point on the instrument case as shown in Fig. 1. A rigid arm which represents the gimbal member is attached to the free end of the elastic member. Forees $F_{x}$ and $F_{y}$, and a moment $M_{0}$, applied to the rigid arm at a point $R(\sin \psi / \psi)$ from the are center on the are bisector produce the following displacements of that point:

$$
\begin{aligned}
\delta_{x} & \left.=\frac{1}{K_{x}} F_{x} \text { (independent of } F_{y} \text { and } M_{0}\right) \\
\delta_{y} & \left.=\frac{1}{K_{y}} F_{y} \text { (independent of } F_{x} \text { and } M_{0}\right), \text { and rotation } \\
a & \left.=\frac{1}{K_{0}} M_{0} \text { (independent of } F_{x} \text { and } F_{y}\right)
\end{aligned}
$$

where

$$
\begin{aligned}
& \frac{1}{K_{x}}=\frac{2 R^{3}}{E I}\left(\frac{\psi}{2}+\frac{\sin 2 \psi}{4}-\frac{\sin ^{2} \psi}{\psi}\right) \\
& \frac{1}{K_{y}}=\frac{2 R^{3}}{E I}\left(\frac{\psi}{2}-\frac{\sin 2 \psi}{4}\right) \\
& \frac{1}{K_{0}}=\frac{2 R \psi}{E I}
\end{aligned}
$$

and it is assumed that the values of $\delta / R, h / R$, and $a / \psi$ are all much less than unity.

The point of application of $F_{x}$ and $F_{y}$ is called the "elastic center." The torsional stiffness, or spring rate of the elastic member for rotation about any point other than the elastic center, is larger than $K_{0}$, as shown below. Minimum spring rate then occurs when the axis of the gimbal member passes through the elastic center.

A simplified method for computing the torsional spring rate for rotation about any point in the plane of the are is given below. Consider a rotation $a$, of a rigid gimbal member about point $x, y$, as shown in Fig. 2. The rotation is produced by $F_{x}, F_{y}$, and $M_{0}$ acting at the elastic center.

The rotation produces displacements

$$
\delta_{x}=a y, \quad \delta_{y}=-a x, \quad a=a
$$

of the elustic center. These displacements are related to $\boldsymbol{F}_{x}, \boldsymbol{F}_{y}$, and $M_{0}$ as follows: 


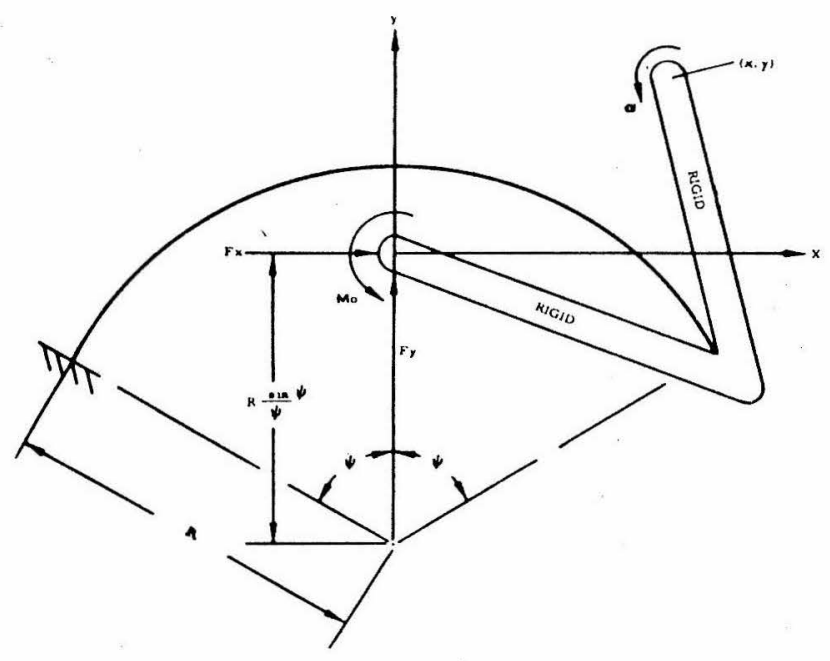

Fig. 2 Application of forces to an elastic member by a gimbal member pivoted at a point other than the elastic center

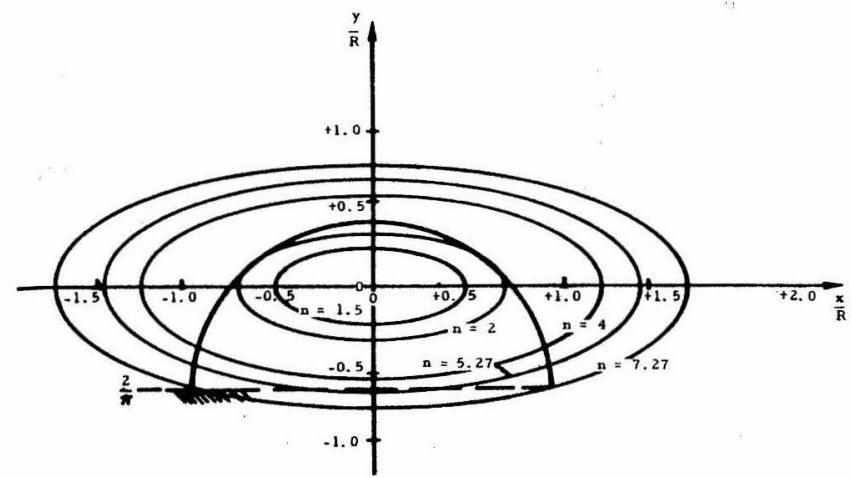

Fig. 3 Trajectories of $n=K_{x y} / K_{0}$ for several rotation centers, for the special case $\psi=\pi / 2$

$$
F_{x}=K_{x} a y, \quad F_{y}=-K_{y} a x, \text { and } M_{0}=K_{0} a .
$$

The same displacements are produced by applying a statically equivalent set of reactions $F_{x}, F_{y}$, and $M_{x y}$ to the rigid gimbal member at the point of rotation $(x, y)$, where $M_{x y}=M_{0}+F_{x} y-$ $F_{y} x$, or $M_{x y}=K_{0} a+K_{x} a y^{2}+K_{y} a x^{2}$. The torsional spring rate about $x, y$ is then $M_{x y} / a$, or

$$
K_{x y}=K_{0}+K_{x} y^{2}+K_{y} x^{2} .
$$

The minimum spring rate occurs for rotation about the elastic center $x=y=0$. Equation (2) may be used to calculate the torsional spring rate about any point $x, y$ after evaluation of the constants $K_{0}, K_{x}$, and $K_{y}$, equation (1) [for a particular member of radius $R$, length $2 R \psi$, and flexural rigidity $E I]$.

Centers of rotation which lie on the ellipse

$$
\frac{x^{2}}{\frac{K_{0}(n-1)}{K_{y}}}+\frac{y^{2}}{\frac{K_{0}(n-1)}{K_{x}}}=1
$$

have a torsional spring rate $K_{x y}=n K_{0}$. When $K_{x}=K_{v}(\sin \psi$ $=0, \psi=\tan \psi$ ), the torsional spring rate about any point on a circle of radius $r=\left[K_{0}(n-1) / K_{x}\right]^{1 / 2}$ with its center at the elastic center is $K_{x y}=n K_{0}$.

Values of $n=K_{x y} / K_{0}$ for several rotation centers are shown in Fig. 3 for the special case wherein

$$
\psi=\frac{\pi}{2}, \frac{1}{K_{0}}=\frac{\pi R}{E I}, \frac{K_{x}}{K_{0}}=\frac{\pi}{\left(\frac{\pi}{2}-\frac{4}{\pi}\right) R^{2}}, \quad \frac{K_{\nu}}{K_{0}}=\frac{2}{R^{2}} .
$$

\section{Acknowledgment}

Acknowledgment is due to Messrs. J. M. Slater and W. A. Ebert of Autoneties Division, North American Aviation, Inc., for suggesting the investigation reported herein and for helpful discussions.

\section{Steady-State Response of Linear Vibration Systems With Viscous Damping}

\section{MORTON E. GURTIN}

The basic matrix ${ }^{2}$ equation for forced vibration is:

$$
M \ddot{q}+C \dot{q}+K_{q}=f e^{j \omega t}
$$

Guderley ${ }^{3}$ demonstrates that any $n$th order equation of this form may be transformed into one of the first order by a suitable change of co-ordinates. In the present case, the following form suggested by Foss, ${ }^{4}$ as well as by other investigators ${ }^{5,6,7}$, is used.

$$
B \dot{z}+D z=f^{\prime} e^{j \omega t},
$$

where

$$
\begin{gathered}
B=\left[\begin{array}{ll}
0 & M \\
M & C
\end{array}\right], \quad D=\left[\begin{array}{ll}
-M & 0 \\
0 & K
\end{array}\right], \\
z=\left\{\begin{array}{l}
\dot{q} \\
q
\end{array}\right\}, \quad f^{\prime}=\left\{\begin{array}{l}
0 \\
f
\end{array}\right\}, \\
j=\sqrt{-1}
\end{gathered}
$$

The homogeneous solution will be of the form

$$
z=l e^{\alpha t},
$$

and the homogeneous equation then gives

$$
\alpha B l+D l=0 .
$$

If $N$ was the original number of degrees of freedom, then (5) will yield $2 N$ eigenvectors and eigenvalues

${ }^{1}$ General Engineering Laboratory, General Electric Company, Schenectady, N. Y.

2 The following matrix nomenclature will be used: An upper-case letter will denote a matrix, and a lower-case letter a vector; a $t$ superscript will denote the transpose.

${ }^{3}$ K. G. Guderley, "On Nonlinear Eigenvalue Problems of Matrices," Journal of the Society for Industrial and Applied Mathematics, vol. 6, December, 1958, pp. 335-353.

$4 \mathrm{~K}$. A. Foss, "Co-Ordinates Which Uncouple the Equations of Motion of Damped Linear Dynamic Systems," Journal of Applied Mechanics, vol. 25, Trans. ASME, vol. 80, 1958, pp. 361-364.

5 F. L. Bauer, "Zur numerischen Behandlung von algebraischen Eigenwertproblemen hoherer Ordnung," Zeitschrift für Anrewandte Mathematik und Mechanik, vol. 36, 1956, pp. 244-245.

- J. Morris, "Linear Simultaneous Equations and Cognate Quadratic Forms," Report No. SME 4039, Royal Aircraft Establishment, Farnsborough, England.

7 W. J. Duncan and A. R. Collar, "Matrices Applied to the Motions of Damped Systems," Philosophical Magazine, series 7, vol. 19, 1935, p. 197.

Manuscript received by ASME Applied Mechanics Division, February 2, 1959. 